\title{
Assessment of Oral Health-related Quality of Life in Patients Suffering from Systemic Diseases
}

\author{
Kavita Dhinsa ${ }^{1}$, Sonali Saha ${ }^{2}$, Abhay M Tripathi ${ }^{3}$, Gurpreet Dhinsa ${ }^{4}$, Vivek Rai ${ }^{5}$, Jitendra Bhagchandani ${ }^{6}$
}

\begin{abstract}
Aim: To assess the oral health-related quality of life (OHRQoL) among children suffering from congestive heart failure and bronchial asthma in Lucknow city.

Materials and method: Methodology: Patients aged 6-12 years were assessed using Child Perception Questionnaire (CPQ). DMFT was assessed in same patients to measure their caries experience.

Results: Dental caries were observed in $62.6 \%$ of cardiac patients followed by $55.8 \%$ in bronchial asthma patients.

Conclusion: Children with CHF had high dental caries experience as compared to asthmatic patients. Due to high caries exposure, they had a negative impact on OHRQoL as compared to others.

Clinical significance: The relationship between oral and systemic health must be consistently reinforced to a patient and guardians of children with systemic disease that can enable to improve the quality of life of these compromised populations.

Keywords: Bronchial asthma, Cardiac patients, DMFT, Oral health, Quality of life.

International Journal of Clinical Pediatric Dentistry (2021): 10.5005/jp-journals-10005-2079
\end{abstract}

\section{INTRODUCTION}

Considering oral health, early childhood caries is the commonest disease among children. It can affect the oral health of a child, which in turn affects the quality of life of Pediatric patients. It expresses the insight of an individual relating their state of mind, their cultural and system of values in which they live, their expectations, standard, objectives, and concerns. On the other hand, oral health quality of life explains the subjective acuity of an individual based on the impact of the disease and its treatment. ${ }^{1}$

In recent years, much attention has been focused on children with systemic diseases such as cardiovascular dysfunction, bronchial asthma, etc., who have difficulties in maintaining and performing efficient oral hygiene thereby contributing to a higher risk of diseases related to odontogenic origin. As such patients' influence of poor oral health is seen in their daily life which can be judged by evaluating their Oral Health-related quality of life (OHRQoL). ${ }^{2}$ A biopsychosocial model of health comprising of social and emotional well-being of an individual along with symptoms and physical functioning is incorporated in OHQoL. ${ }^{3}$ Healthcare professionals other than dentists are not aware of the risks and harmful effects of dental disease for children with systemic disease or they may be distracted by the various challenges associated with caring for a child with systemic diseases such that oral care is neglected. ${ }^{4}$ Hence, keeping in mind all the above factors, this research was carried out to assess the oral health-related quality of life among the pediatric population suffering from congestive heart failure and bronchial asthma in Lucknow city.

\section{Materials and Methodology}

This present study was conducted in the Department of Pedodontics \& Preventive Dentistry in collaboration with the
1-3,5 Department of Pediatrics and Preventive Dentistry, Sardar Patel Post Graduate Institute of Dental and Medical Sciences, Lucknow, Uttar Pradesh, India

${ }^{4}$ Department of Periodontology, Career Institute of Dental Sciences \& Hospital, Lucknow, Uttar Pradesh, India

${ }^{6}$ Department of Orthodontics and Dentofacial Orthopedics, Sardar Patel Post Graduate Institute of Dental and Medical Sciences, Lucknow, Uttar Pradesh, India

Corresponding Author: Kavita Dhinsa, Department of Pediatrics and Preventive Dentistry, Sardar Patel Post Graduate Institute of Dental and Medical Sciences, Lucknow, Uttar Pradesh, India, Phone: +91 9721222260, e-mail: grover.kavita@gmail.com

How to cite this article: Dhinsa K, Saha S, Tripathi AM, et al. Assessment of Oral Health-related Quality of Life in Patients Suffering from Systemic Diseases. Int J Clin Pediatr Dent 2021;14(6):779-783.

Source of support: Nil

Conflict of interest: None

Department of Cardiology and Pulmonary Medicine, Sanjay Gandhi Postgraduate Institute of Medical Sciences, Lucknow.

\section{Test Groups}

A total of 180 Pediatric patients between 8 and 16 years were chosen for the study, which were divided into three groups as follows:

- Group 1: Patients diagnosed with Bronchial Asthma

- Group 2: Patients diagnosed with Congestive Heart Failure

- Group 3: Patients free of any disease (control).

Ethical committee clearance and informed consent were obtained and confidentiality of participants was strictly maintained.

Patients whose parents or caregivers gave consent and those who were physically present were included in the study whereas children who were anxious and apprehensive and with special healthcare needs were excluded from the study.

\footnotetext{
(c) The Author(s). 2021 Open Access This article is distributed under the terms of the Creative Commons Attribution 4.0 International License (https://creativecommons. org/licenses/by-nc/4.0/), which permits unrestricted use, distribution, and non-commercial reproduction in any medium, provided you give appropriate credit to the original author(s) and the source, provide a link to the Creative Commons license, and indicate if changes were made. The Creative Commons Public Domain Dedication waiver (http://creativecommons.org/publicdomain/zero/1.0/) applies to the data made available in this article, unless otherwise stated.
} 


\section{Oral Health Assessment}

Two-staged calibration was done:

\section{- Theoretical stage}

- First section: Gathering of demographic data including name, age, gender, frequency of brushing and dental visits, and being referred to a dentist by a physician.

- Second section: Child Perception Questionnaire $\left(\mathrm{CPQ}_{11-14}\right)$ proposed by Jokovic et al. (2002) was used to record the patient's insights about the importance of oral health and accessibility of such care. ${ }^{5}$ This questionnaire is comprised of four domains relating to the quality of life:

- Emotional well-being

- Oral symptoms related to disease

- Normal functioning of individual

- Social well-being

All above domains had questions related to their relation and frequency of events as applied to teeth, lips, jaws, and mouth in the previous three months. Assessment of score was done using Likert's scale ( 1 to 4 ), wherein $1=$ sometimes; 2 = often; $3=$ everyday and $4=$ almost everyday. The total $C \mathrm{PQ}_{11-14}$ score was obtained by adding all of the item scores under each domain. The total score ranges from 0 to 148, a higher score means a greater degree of the impact of oral conditions on the quality of life of a child.

\section{- Clinical Examination}

After recording of survey for the study population, oral and dental examination was performed in which DMFT status was assessed using WHO criteria, representing the effect of disease. All relevant clinical findings found during examination were conversed with the guardian and those requiring dental treatments were referred for care. Oral health education regarding the importance of oral hygiene, dental caries, etc., was also discussed with the patient and guardians. All the data was collected tabulated and sent for statistical analysis.

\section{Statistical Analysis}

Estimation of sample size was done by using G Power software (version 3.0) for $F$ test and ANOVA: Omnibus fixed one way, for three groups was chosen. A sample size of 180 (60 per group) was found to be sufficient for an alpha of 0.05 , power of $80 \%$, 0.25 as effect size (assessed for difference in OHRQoL assessed by $(P Q)$. Data were analyzed using SPSS version 21. Categorical and Continuous variables were summarized as absolute and amp; relative frequencies and Means and amp; SD respectively, respectively. Graphs were prepared on Microsoft Excel. One way
ANOVA test was done for Intergroup comparison and the level of statistical significance was set at 0.05 .

\section{Results}

Table 1 and Figure 1 depict that amongst 180 samples, 101 were females and 79 were males who participated in the survey. The distribution of males and females was not found to be significantly different among study groups.

Table 2 and Figure 2 stated that the mean DMFT score was found to be significantly different among study groups. The mean DMFT score of cardiac patients (Group 2) was found to be significantly more than that of bronchial asthma patients (Group 1), which was further significantly more than that of control (Group 3).

Table 3 and Figure 3 stated that the mean Oral Symptoms (OS) domain, Functional (FL) domain, Social Well-being (SWB) domain and Emotional Well-being (EWB) domain scores, were found to be significantly different among study groups. The Mean OS domain, FL domain, SWB domain and EWB domain scores of Group $2(87.7 \pm 11.27)$ was found to be significantly more than that of Group 1 ( $56.6 \pm 20.5)$ which was further significantly more than that among Group 3 (40.85 \pm 19.9).

\section{Discussion}

In the past 3 years, a psychosocial global model of quality of life that evolves from various domains of life has been developed

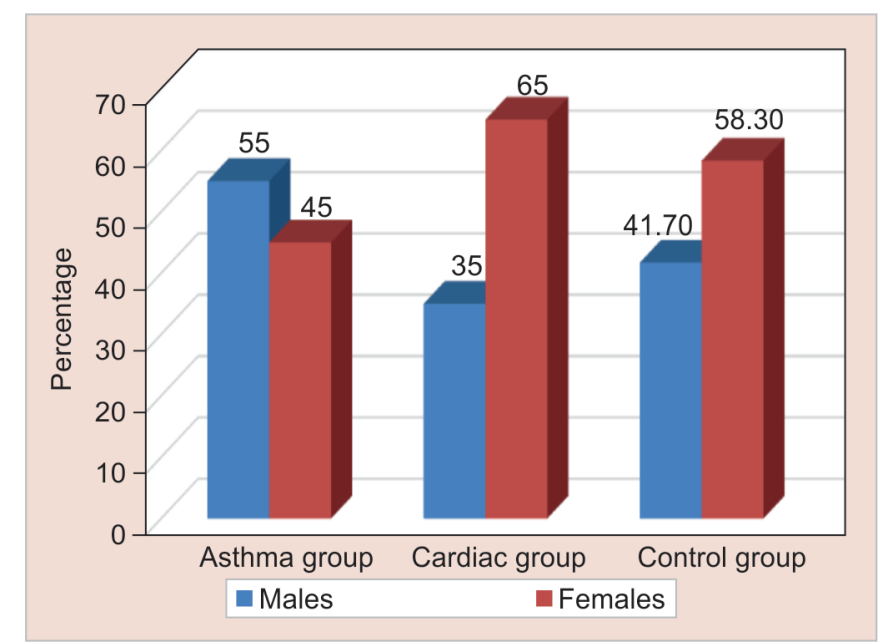

Fig. 1: Distribution of sample size

Table 1: Distribution of study samples

\begin{tabular}{|c|c|c|c|c|c|}
\hline & & & \multicolumn{2}{|l|}{ Sex } & \multirow{2}{*}{ Total } \\
\hline & & & Males & Females & \\
\hline \multirow[t]{6}{*}{ Group } & Asthma & $\mathrm{N}$ & 33 & 27 & 60 \\
\hline & & $\%$ & $55.0 \%$ & $45.0 \%$ & $100.0 \%$ \\
\hline & Cardiac & $\mathrm{N}$ & 21 & 39 & 60 \\
\hline & & $\%$ & $35.0 \%$ & $65.0 \%$ & $100.0 \%$ \\
\hline & Control & $\mathrm{N}$ & 25 & 35 & 60 \\
\hline & & $\%$ & $41.7 \%$ & $58.3 \%$ & $100.0 \%$ \\
\hline \multirow[t]{2}{*}{ Total } & & $\mathrm{N}$ & 79 & 101 & 180 \\
\hline & & $\%$ & $43.9 \%$ & $56.1 \%$ & $100.0 \%$ \\
\hline$p$ value & & & 0.08, NS & & \\
\hline
\end{tabular}


and used quite extensively in medical research to assess the individual's perception of overall wellbeing. Since many systemic diseases affect the lives of children and their caregivers, Quality of life is often used as an outcome to describe how a child's systemic disease is impacting his/her daily life. Assessment of OHRQoL measures a different component of oral health status than other clinical measures. ${ }^{3}$ Likewise other measures like stress and anxiety produced due to dental diseases, measurement of quality of life is also important as it can influence the adulthood of the patient. ${ }^{6}$ Therefore, this research was planned to evaluate the quality of life in patients suffering from cardiac disease and bronchial asthma.

Oral health quality of life is a concept that relies on a patient's awareness. Since in the pediatric population, teeth and facial development vary markedly with age and also the understanding of basic health concepts may be challenging in younger age-group therefore, children between 8 and 16 years were considered. The age of 8 years marks the beginning of abstract thinking and self-concept, like the subjects of this study. Patients with systemic diseases often exhibit negative behavioral patterns during dental treatment due to the previous experiences in the medical hospital Due to cognitive impairment; it is difficult to understand their cognitive processes, and consequently results in an unreliable measurement of QoL. Therefore, studies on pediatric patients OHRQoL rely on the awareness of their primary caregivers. ${ }^{7}$

Assessment of OHRQoL was done using the Child Perception Questionnaire $\left(\mathrm{CPQ}_{11-14}\right)$, which is reliable, sensitive to children's emotional \& Social development. It not only evaluates the impact on oral health problems but also throws light on child's personal physical, psychological, emotional \& behavioral characteristics. ${ }^{5}$
This questionnaire has been previously used by Page et al., Bennadi and Reddy, and Alzoubi et al..$^{8-10}$

Results of the present study stated that Mean DMFT score was found to be significantly different among study groups. The mean DMFT score of Group 2 (Bronchial Asthma) was found to be significantly more than that of Group 1 (Cardiac patients) which was further significantly more than that among Group 3 (Control).

Results were in accordance with Thomas et al., Bertoletti et al., Nair et al., Yadav et al., Andre et al., Noushadali et al., and Raj et al. ${ }^{11-17}$

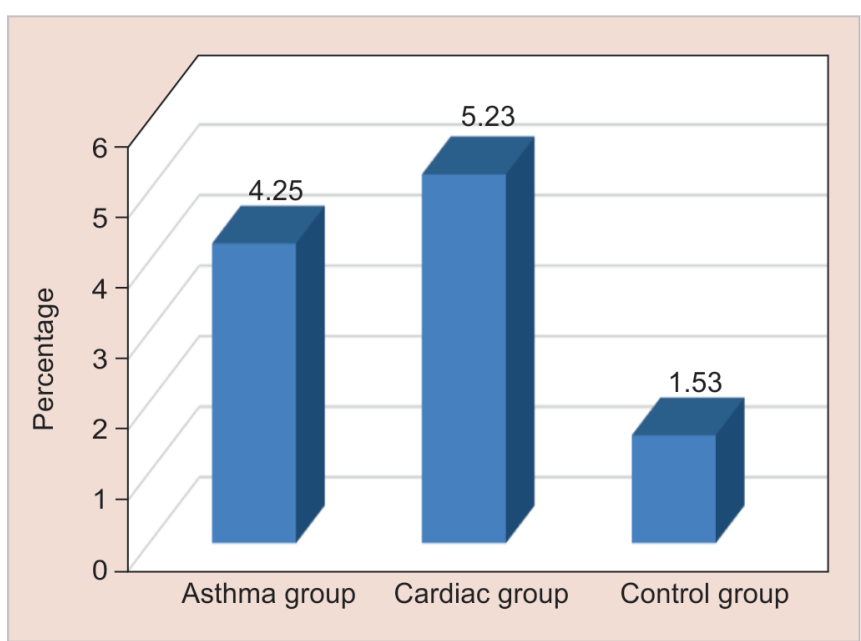

Fig. 2: Mean DMFT score in tested groups

Table 2: Mean DMFT score of sample groups

\begin{tabular}{|c|c|c|c|c|c|c|}
\hline & & $N$ & Mean & Std. deviation & $p$ value & Post hoc pairwise comparison \\
\hline \multirow[t]{3}{*}{ DMFT } & 1 & 60 & 4.25 & .773 & $<0.0001, \mathrm{~S}$ & Gr $2>\operatorname{Gr} 1>$ Gr 3 \\
\hline & 2 & 60 & 5.23 & 1.978 & & \\
\hline & 3 & 60 & 1.53 & 1.157 & & \\
\hline
\end{tabular}

Table 3: Mean Oral Symptoms (OS) domain, Functional (FL) domain, Social Well-being (SWB) domain, and Emotional Well-being (EWB) domain scores amongst tested samples

\begin{tabular}{|c|c|c|c|c|c|c|}
\hline & & $N$ & Mean & Std. deviation & $p$ value & $\begin{array}{l}\text { Post hoc pairwise com- } \\
\text { parison }\end{array}$ \\
\hline \multirow[t]{3}{*}{ os } & Gr 1 & 60 & 11.3333 & 4.10319 & $<0.0001$ & Gr $2>\operatorname{Gr} 1>\operatorname{Gr} 3$ \\
\hline & Gr 2 & 60 & 17.8833 & 2.31496 & & \\
\hline & Gr 3 & 60 & 8.4167 & 4.55584 & & \\
\hline \multirow[t]{3}{*}{$\mathrm{FL}$} & Gr 1 & 60 & 11.3333 & 4.10319 & $<0.0001$ & Gr $2>\operatorname{Gr} 1>\operatorname{Gr} 3$ \\
\hline & Gr 2 & 60 & 17.1833 & 2.47353 & & \\
\hline & Gr 3 & 60 & 8.2833 & 4.25896 & & \\
\hline \multirow[t]{3}{*}{ EWB } & Gr 1 & 60 & 13.6000 & 4.92383 & $<0.0001$ & Gr $2>\operatorname{Gr} 1>\operatorname{Gr} 3$ \\
\hline & Gr 2 & 60 & 21.1333 & 3.02233 & & \\
\hline & Gr 3 & 60 & 9.6833 & 4.55658 & & \\
\hline \multirow[t]{3}{*}{ SWB } & Gr 1 & 60 & 20.4000 & 7.38574 & $<0.0001$ & Gr $2>\operatorname{Gr} 1>$ Gr 3 \\
\hline & Gr 2 & 60 & 31.5000 & 3.82454 & & \\
\hline & Gr 3 & 60 & 14.4833 & 6.94895 & & \\
\hline \multirow[t]{3}{*}{ CPQ score } & Gr 1 & 60 & 56.6667 & 20.51594 & $<0.0001$ & Gr $2>\operatorname{Gr} 1>\operatorname{Gr} 3$ \\
\hline & Gr 2 & 60 & 87.7000 & 11.27664 & & \\
\hline & Gr 3 & 60 & 40.8667 & 19.91717 & & \\
\hline
\end{tabular}




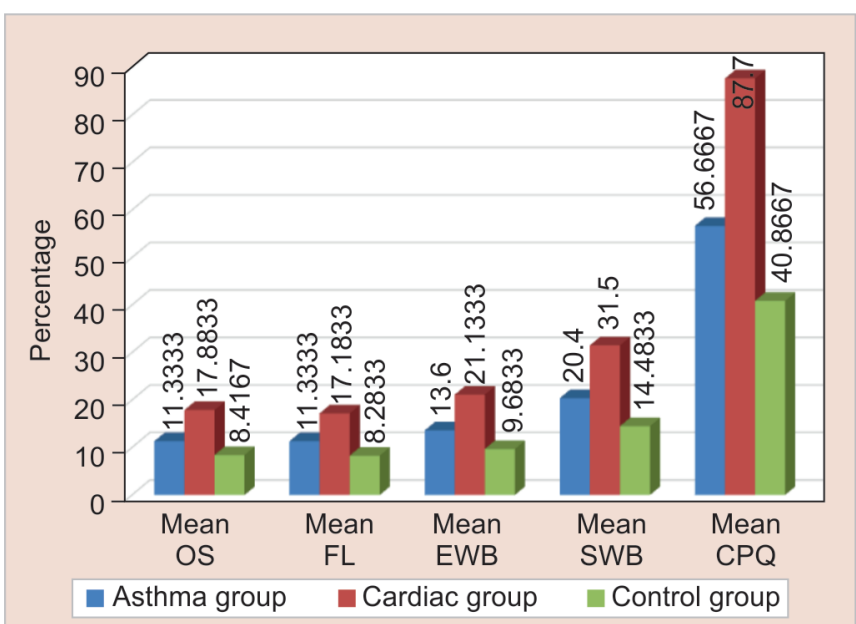

Fig. 3: Mean score of all domains of quality of life

The results were attributed to several factors:

- High frequency of developmental defects increases susceptibility to caries.

- Frequent vomiting due to nutritional problems.

- Lack of oral hygiene due to sweetened medications \& nutritional supplements.

- Medications-induced Xerostomia.

- Cleanliness difficulties due to gingival hypertrophy lead to poor oral hygiene and dental caries.

- Low priority is given to the maintenance of oral care.

- Little knowledge about oral health. ${ }^{18}$

Results of the present study also stated that DMFT in Group 2 (bronchial asthma) was high as compared to Group 3 (control group). This could be due to the fact that patients with asthma have a different immune response and during an episode of rhinitis or an attack, they tend to develop mouth breathing habit leading to serious oral health problems [Yadav et al.]..$^{14}$

Also, Arafa et al. observed that there is decreased salivary and plaque $\mathrm{pH}$ in asthmatic patients using inhalers. ${ }^{19}$ As stated by Dubey et al. the management of asthma patients involves $\beta$-2-adrenoceptor agonists which cause impaired salivary secretion thereby increasing caries susceptibility. According to him, there are presence of specific auto-antibodies to $\beta$-2-adrenoceptor agonists that alters the salivary flow and composition. ${ }^{20}$

Results of the present study showed that mean OS domain, FL domain, SWB domain and EWB domain scores were found to be significantly different among study groups. The Mean OS domain, FL domain, SWB domain and EWB domain scores of Group 2 (bronchial asthma) was found to be significantly more than that of Group 1 (Cardiac patients) which was further significantly more than that among Group 3 (control group).

Results were in accordance with da-Fonseca et al., Miadich et al., and El-Gilany et al. ${ }^{4,21,22}$

This variation could be attributed to failure to prevent dental caries, thereby adding to health problems. Also, the use of GA to treat extensive carious lesions creates more of physical, emotional, and financial burden. Since prevention of caries requires proper dietary habits and oral hygiene care, which can be overwhelming to the families. ${ }^{4}$

Results of the present study showed that Mean OS domain, FL domain, SWB domain and EWB domain scores were found to be significantly different among study groups. The Mean OS domain, FL domain, SWB domain and EWB domain scores of Group 1 (Cardiac patients) was found to be significantly more than that of Group 3 (control group).

This could be due to the fact that a common reason for going to the hospital is asthma in childhood, along with poverty and ethnic minority. Also, altered immune response in such patients along with undesired repair of damaged airways, longterm inflammation leading to altered adult paths. ${ }^{15}$

\section{Conclusion}

The recent transformation from a complete dental approach to a bio-psychosocial-dental approach, $\mathrm{OHQ}$ L plays an important role in oral health-related research. The relationship between systemic diseases and oral health must be consistently reinforced to a patient and guardians, as the most significant barrier to good oral health is often a lack of knowledge. Further researches are needed in this domain that can enable to recover the quality of life of these compromised populations.

\section{References}

1. Sertcelik T, Alkan F, Sapmaz SY, et al. Life quality of children with congenital heart diseases. Turk Arch Pediatr 2018;53:78-86. DOI: 10.5152/turkpediatriars.2018.6428

2. Akhter R, Hassan NMM, Martin EF, et al. Caries experienceand oral health-related quality of life (OHQoL) of children and adoloscents with cerebral palsy in a low-resource setting. BMC Oral Health 2019; 19:1-16. DOI: 10.1186/s12903-018-0704-2

3. Locker D, Jokovic A, Clarke M. Assessing the responsiveness of measures of oral health-related quality of life. Community Dent Oral Epidemiol 2004;32:10-18. DOI: 10.1111/j.1600-0528.2004.00114.x

4. da Fonseca MA, Evans M, Teske D, et al. The impact of oral health on the quality of life of young patients with congenital cardiac disease Cardiol Young 2009;19:252-256. DOI: 10.1017/S1047951109003977

5. Jokovic A, Locker D, Stephens M, et al. Validity and reliability of a questionnaire for measuring child oral-health-related quality of life J Dent Res 2002;81(7):459-463. DOI: 10.1177/154405910208100705

6. Dhinsa K, Saha S, Singh G, et al. Assessment and comparison of dental anxiety by measuring physiological, psychological and immune responses in pediatric patients undergoing Noninvasive dental treatment. J South Asian Assoc Ped Dent 2019;2(1):14-21. DOI: 10.5005/jp-journals-10077-3023

7. Song J, Hyun HK, Shin TJ, et al. Effects of dental treatment and systemic diseaseon oral health-relatedquality of life in Korean pediatric patients. BMC Oral Health 2018;18:1-9. DOI: 10.1186/s12903-018-0552-0

8. Page LAF, Thomson WM, Jokovic $A$, et al. Validation of the child perceptions questionnaire $\left(\mathrm{CPQ}^{11-14}\right)$. J Dent Res 2005;83(9):649-652. DOI: 10.1177/154405910508400713

9. Bennadi D, Reddy CVk. Oral health-related quality of life. J Int Soc Prev Community Dent 2013; 3(1): 1-6. DOI: 10.4103/2231-0762.115700

10. Alzoubi EE, Attard NJ. Oral health-related quality of life impact in dentistry. Res Rev J Dent Sci 2017;5(2):35-42. DOI: 10.15406/ jdhodt.2017.06.00221

11. Thomas MS, Parolia A, Kundabala $M$, et al. Asthma and oral health: a review. Aust Dent J 2010;55:128-133. DOI: 10.1111/j.18347819.2010.01226.x

12. Nair S, Nair S, Sundaram S. A prospective study to assess the quality of life in children with asthma using the pediatric asthma quality of life questionnaire. Ind J Allerg Asthma Immunol 2014;28(1):13-18. DOI: 10.4103/0972-6691.134210

13. Andre A, Pierre GC, MCAndrew M. Quality of life among dental students: a survey study. J Dent Edu 2017;81(10):1164-1169. DOI: 10.21815/JDE.017.074 
14. Yadav PK, Saha S, Singh S, et al. Oral health status and treatment needs of asthmatic children aged 6-12 years in Lucknow. J Ind Assoc Pub Health Dent 2017;15:122-126. DOI: 10.4103/jiaphd.jiaphd_136_16

15. Bertoletti J, Marx GC, Junior SPH, et al. Quality of life and congenital heart disease in childhood and adolescence. Arq Bras Cardiol 2014; 102(2):192-198. DOI: 10.5935/abc.20130244

16. Noushadali AK, Mohandas U, Hussain T. Assessment of health-related quality of life in pediatric asthma patients. Int J Contemp Pediatr 2018; 5(4):1255-1260. DOI: 10.18203/2349-3291.ijcp20182078

17. Raj M, Sudhakar A, Roy R, et al. Health-related quality of life (HRQOL) in children and adolescents with congenital heart disease: a cross- sectional survey from South India. BMJ Paediatr Open 2019;3(1):e000377. DOI: 10.1136/bmjpo-2018-000377

18. Carrillo C, Russell J, Judd P, et al. Oral health of children with congenital heart disease at a pediatric health science centre. J Can Dent Assoc 2018;84:1-5.
19. Arafa A, Aldahlawi S, Fathi A. Assessment of the oral health status of asthmatic children. Eur J Dent 2017;11(3):357-363. DOI: 10.4103/ejd. ejd_65_17

20. Dubey S, Saha S, Tripathi AM, et al. A comparative evaluation of dental caries status and salivary properties of children aged 5-14 years undergoing treatment for acute lymphoblastic leukaemia, type I diabetes mellitus and asthma - in vivo. J Ind Soc Ped Prev Dent 2018;36(3):283-289. DOI: 10.4103/JISPPD. JISPPD_46_18

21. Miadich SA, Everhart RSE, Borschuk AP, et al. Quality of life in children with asthma: a developmental perspective. J Ped Psychol 2015;40(7): 672-679. DOI: 10.1093/jpepsy/jsv002

22. El-Gilany AH, Desoky TE, El-Hawary AK, et al. Quality of life of children with bronchial asthma and their caregivers: a hospital-based study. Prog Med Science 2018;2(1):1-8. DOI: 10.5455/pms.20180419090958 